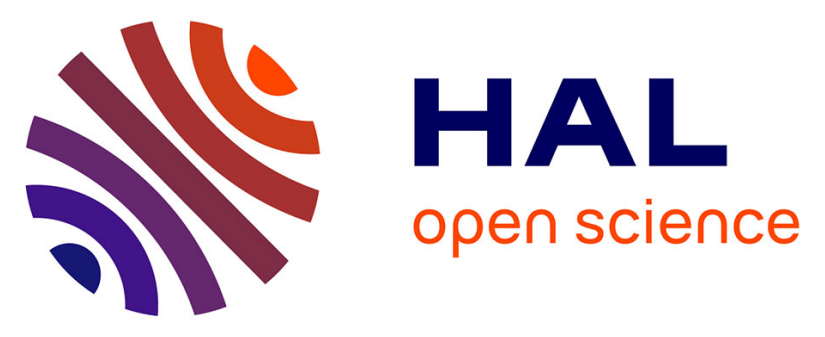

\title{
Transcranial Sonographic Assessment of the Third Ventricle in Neuro-ICU Patients to Detect Hydrocephalus: A Diagnostic Reliability Pilot Study
} Remy Widehem, Paul Bory, Frederic Greco, Frederique Pavillard, Kevin Challard, Alexandre Mas, Flora Djanikian, Julie Carr, Nicolas Molinari, Samir Jaber, et al.

\section{To cite this version:}

Remy Widehem, Paul Bory, Frederic Greco, Frederique Pavillard, Kevin Challard, et al.. Transcranial Sonographic Assessment of the Third Ventricle in Neuro-ICU Patients to Detect Hydrocephalus: A Diagnostic Reliability Pilot Study. 2021. hal-03090815

\section{HAL Id: hal-03090815 https://hal.science/hal-03090815}

Preprint submitted on 2 Aug 2021

HAL is a multi-disciplinary open access archive for the deposit and dissemination of scientific research documents, whether they are published or not. The documents may come from teaching and research institutions in France or abroad, or from public or private research centers.
L'archive ouverte pluridisciplinaire HAL, est destinée au dépôt et à la diffusion de documents scientifiques de niveau recherche, publiés ou non, émanant des établissements d'enseignement et de recherche français ou étrangers, des laboratoires publics ou privés. 


\title{
Transcranial sonographic assessment of the third ventricle in neuro-ICU patients to detect hydrocephalus: a diagnostic reliability pilot study
}

\author{
Rémy Widehem ${ }^{1}$, Paul Bory ${ }^{1}$, Frédéric Greco ${ }^{1}$, Frédérique Pavillard ${ }^{1}$, Kévin Chalard ${ }^{1}$, Alexandre Mas ${ }^{1}$, \\ Flora Djanikian', Julie Carr², Nicolas Molinari ${ }^{3}$, Samir Jaber ${ }^{2,4}$, Pierre-François Perrigault ${ }^{1}$ and \\ Gerald Chanques ${ }^{1,2,4^{*}}$ (D)
}

\begin{abstract}
Background: Transcranial sonography is a point-of-care tool recommended in intensive care units (ICU) to monitor brain injured patients. Objectives of the study was to assess feasibility and reliability of the third ventricle (V3) diameter measurement using transcranial sonography (TCS) compared to brain computed-tomography (CT), the gold standard measurement, and to measure the TCS learning curve. Design: prospective study, in a 16-bed neurological ICU in an academic hospital. Every consecutive brain injured adult patient, who required a brain CT and TCS monitoring were included. The V3 diameter was blindly measured by TCS and CT. Intraclass correlation coefficient (ICC) and Bland-Altman plot were used to assess the reliability and agreement between TCS and CTV3 measurements. Diagnosis performance of the V3 diameter using TCS to detect hydrocephalus was measured. Absolute difference between V3 measurement by residents and experts was measured consecutively to assess the learning curve.
\end{abstract}

Results: Among the 100 patients included in the study, V3 diameter could be assessed in 87 patients (87\%) from at least one side of the skull. Both temporal windows were available in 70 patients (70\%). The ICC between V3 diameter measured by TCS and CT was 0.90 [95\% Cl 0.84-0.93] on the right side, and 0.92 [0.88-0.95] on the left side. In Bland-Altman analysis, mean difference, standard deviation, 95\% limits of agreement were $0.36,1.52,-2.7$ to $3.3 \mathrm{~mm}$, respectively, on the right side; $0.25,1.47,-2.7$ to $3.1 \mathrm{~mm}$, respectively, on the left side. Among the 35 patients with hydrocephalus, V3 diameters could be measured by TCS in 31 patients (89\%) from at least one side. Hydrocephalus was, respectively, excluded, confirmed, or inconclusive using TCS in 35 (40\%), 25 (29\%) and 27 (31\%) of the 87 assessable patients. After 5 measurements, every resident reached a satisfactory measurement compared to the expert operator.

Conclusion: TCS allows rapid, simple and reliable V3 diameter measurement compared with the gold standard in neuro-ICU patients. Aside from sparing irradiating procedures and transfers to the radiology department, it may especially increase close patient monitoring to detect clinically occult hydrocephalus earlier. Further studies are needed to measure the potential clinical benefit of this method.

Trial registration: ClinicalTrials.gov ID: NCT02830269.

\footnotetext{
*Correspondence: g-chanques@chu-montpellier.fr

${ }^{1}$ Department of Anaesthesia \& Critical Care Medicine, Montpellier

University Hospital Center, Gui de Chauliac Hospital, Montpellier, France

Full list of author information is available at the end of the article
} adaptation, distribution and reproduction in any medium or format, as long as you give appropriate credit to the original author(s) and the source, provide a link to the Creative Commons licence, and indicate if changes were made. The images or other third party material in this article are included in the article's Creative Commons licence, unless indicated otherwise in a credit line to the material. If material is not included in the article's Creative Commons licence and your intended use is not permitted by statutory regulation or exceeds the permitted use, you will need to obtain permission directly from the copyright holder. To view a copy of this licence, visit http://creativeco mmons.org/licenses/by/4.0/. 
Keywords: Neurocritical care, Third ventricle, Sonography, Hydrocephalus, Point of care

\section{Introduction}

Transcranial sonography (TCS) is an important pointof-care diagnostic tool $[1,2]$ recommended in neurological Intensive Care Unit (ICU) patients [3-5]. It is generally used for monitoring patients suffering from subarachnoid haemorrhage [6, 7], severe traumatic brain injury [8] or severe stroke. As TCS is an accessible, nonirradiating, easy to learn and reliable tool [9], it can be repeated several times a day at the bedside in order to assess intracranial hypertension $[10,11]$ and to guide therapeutic decisions, by measuring the Doppler velocity of intracerebral arteries. Moreover, TCS can be used to evaluate cerebral anatomy and pathology [12]. In 1990, Bogdahn et al. described the third ventricle (V3) in 45 of 49 patients free of neurological involvement [13]. Other studies found a good sensitivity and specificity of V3 TCS measurement compared to magnetic resonance imaging or computed-tomography (CT) in non-critically ill patients with neurological diseases [14-17]. One study, published 20 years ago, measured the V3 diameter during episodes of intracranial hypertension in 28 ICU patients [18]. This preliminary study did not validate this method in comparison to the gold standard examination, i.e. computed-tomography (CT).

Obstructive hydrocephalus can occur in subarachnoid haemorrhage patient and in patient with external ventricular drain. V3 study can detect this pathological issue. A retrospective study reported a good correlation between TCS and CT in 15 patients with traumatic brain injury for the measurement of V3 diameter [19]. Feasibility and reliability of the V3 diameter measured by TCS have never been prospectively reported in a large population of neuro-ICU patients. Hence, the primary goal of this study was to determine the feasibility and reliability of TCS compared to brain CT in measuring V3 diameter in neuro-ICU patients. Secondly, the diagnosis performance to detect hydrocephalus was measured. Finally, the learning curve for measuring the V3 diameter by TCS was assessed.

\section{Methods}

The study protocol was approved by the Ethics committee of "Comité de Protection des Personnes Sud-Méditerranée I" (ID RCB: 2016-A00749-42; Protocol Version: March 11, 2016; Consent Version: June 25, 2016), ClinicalTrials.gov ID: NCT02830269. Because the trial used non-invasive procedures along with standard care provided in French ICUs [7, 20], only a verbal consent was required from the patient or relatives, according to the
French law [21]. Additional file 1: Figure S1 shows the study design.

\section{Patients}

The study took place in the 16-bed neuro-ICU of the tertiary University Hospital of Montpellier Gui de Chauliac, France. All consecutive neuro-ICU patients $\geq 18$ years old, admitted to the neuro-ICU with a brain injury, were eligible for enrolment if they underwent a brain CT planned by the bedside physicians, as well as TCS monitoring. Exclusion criteria were: a sonographic assessment of the third ventricle impossible to perform within one hour around a brain CT, or consent disclaimed.

\section{Patient characteristics and treatments}

Patient characteristics, including weight $(\mathrm{kg})$, height $(\mathrm{m})$, body mass index $\left(\mathrm{kg} / \mathrm{m}^{2}\right)$, age, gender, type of brain injury, Glasgow Coma Scale, Simplified Acute Physiology Score 2 (SAPS2) [22], Fisher score [23] for subarachnoid haemorrhage and International Severity Score (ISS) [24] for traumatic brain injury were recorded at the time of ICU admission. At the time of the TCS exam, the following variables were recorded: systolic, diastolic and mean arterial pressures, heart rate, intraventricular or intracranial pressure (if available), body temperature, blood glucose level, oxygen plethysmography saturation, presence or absence of an external ventricular drain (EVD) or a craniotomy, mechanical ventilation parameters, arterial blood gas, natremia and haemoglobinemia. The use of sedative drugs, neuromuscular blocking agents, milrinone, nimodipine and norepinephrine was recorded. Intracranial hypertension was assessed using clinical parameters: anisocoria, mydriasis or Cushing reflex. Patient outcomes were assessed: duration of mechanical ventilation, ICU length of stay, RANKIN score and mortality at ICU discharge.

\section{Feasibility and reliability of TCS compared with brain CT (primary goal)}

The primary goal was the feasibility and the reliability of V3 diameter measurements by TCS compared to brain CT (gold standard).

\section{TCS measurements}

The measurements were performed with the patient in supine position, head $30^{\circ}$ up, using a General Electric* Vivid-q 5-1 MHz cardiac transducer for echocardiography (GE, Boston, USA). The 2D mode was used, along with the colour and pulse waved Doppler. Both sides of 
the head were assessed. Cerebral blood flow velocity and pulsatility indexes were recorded on both middle cerebral arteries. The V3 diameter was measured through the temporal acoustic bone window using a low frequency ( 2 to $4 \mathrm{MHz}$ ) probe within one hour around the brain $\mathrm{CT}$. The V3 was identified as a double hyperechogenic image above the midbrain with the diencephalon on both sides (Fig. 1); the V3 diameter was measured within the hyperechogenic lines. Depth was defined as the measurement between the external bone table and the first wall of V3. Measurements were performed only on optimal image planes, after zooming using the electronic calliper of the ultrasound device. An intensivist, who was trained in TCS (expert operator), measured the V3 diameter and reported the measurement on the Clinical Research Form, blinded to the brain CT measurements. Both sides of the head were assessed. Examination times were recorded.

\section{Brain CT}

The diameters and depth of the V3, as well as bone thickness and density, were independently and retrospectively measured by the same investigator (RW), who was blinded to the TCS measurements and to the clinical data. CT acquisition were helicoidal. Slice thickness were

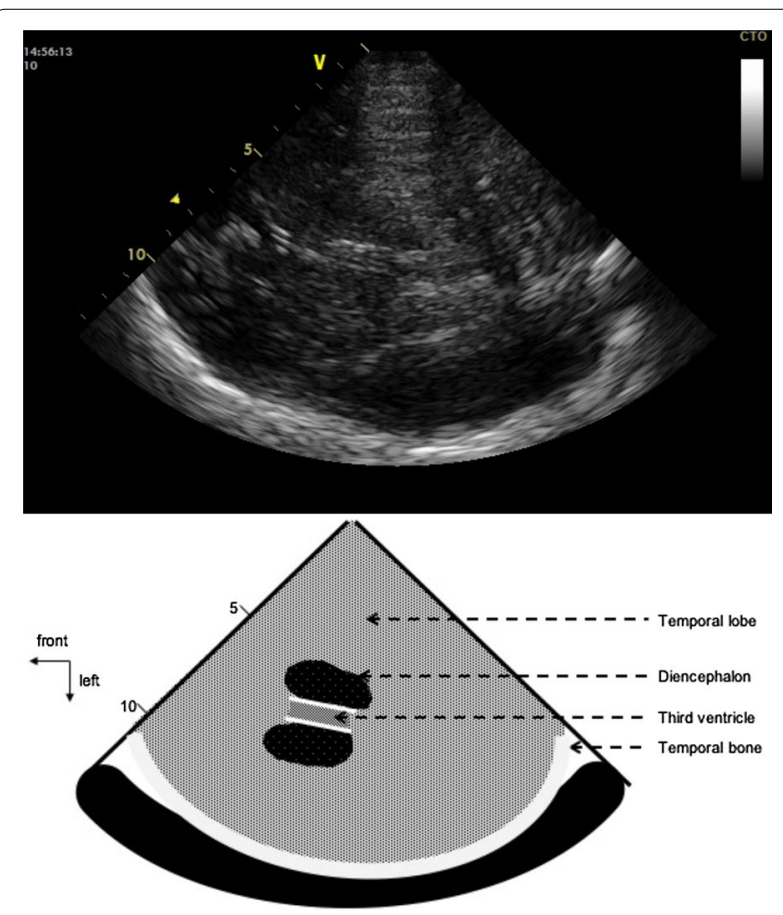

Fig. 1 Sonographic view of $\mathrm{V} 3$ and the corresponding anatomic diagram. The third ventricle was identified as a double hyperechogenic image over the midbrain with the diencephalon on both sides
$1.25 \mathrm{~mm}$ and slice increment $0.625 \mathrm{~mm}$. V3 coronal and transverse diameters were measured, as well as a "modified" diameter, which measured the diameter using the same angulation as classically used for the sonography examination view. "Modified" diameters were measured for both sides. Coronal, transverse and "modified" diameters were all measured in the larger portion of the third ventricle after zooming. Depth was measured between the external temporal bone and the outer limit of the V3. Reconstructions were made manually using coronal view, and measurements were performed on transversal view respecting both anterior/posterior commissure angulation in axial plan [25] and previously measured coronal angulation. Slice thickness and increment remained similar. All reconstructions were performed in the radiology department without files compression and data lost.

\section{Diagnostic performance of V3 measurement using TCS to detect hydrocephalus}

Hydrocephalus was defined by a bicaudal index exceeding the upper limit of normality for the patient's age according to a standardised method on the CT [26, 27]. V3 diameters measured by TCS were analysed secondly to define thresholds and related diagnosis performances characteristics (see "Statistics").

\section{Absolute difference between V3 measurement by residents and experts}

The absolute difference between the V3 measurement by residents and experts was assessed among 9 anaesthesiologist-intensivist residents at another time during the ICU stay. All residents had previous experience in TCS but not in V3 diameter measurement. Residents measured the V3 diameter, followed by an ultrasound expert ICU physician who measured the V3 during the same TCS examination. A quick explanation of the method was performed after each measurement by the expert physician. A learning curve was drawn to represent the evolution of the mean absolute difference between the V3 measurement by the residents and the V3 measurement by an expert physician for consecutive measurements. Reaching $1 \mathrm{~mm}$ of absolute difference between the measurements of the resident and the expert physician was determined to qualify a satisfactory skill. This difference of $1 \mathrm{~mm}$ was determined based on the technical characteristics of the transducer's precision, which is $1 \mathrm{~mm}$ for a 5-1 MHz cardiac transducer using a low frequency (2 to $4 \mathrm{MHz}$ ) at $7 \mathrm{~mm}$ depth.

\section{Statistics}

Data are presented as numbers (percentage) and medians with interquartile range (IQR). The $95 \%$ confidence intervals $(95 \% \mathrm{CI})$ are given when appropriate. 
Feasibility and reliability of TCS compared with brain CT (primary goals)

The feasibility of the measurement of the V3 diameter by TCS was measured as the proportion of assessable patients.

The reliability of TCS in measuring the V3 diameter, compared to the CT measurement considered as the gold standard method [28], was evaluated by the intraclass correlation coefficient (ICC). The Bland-Altman method analysed the agreement between the two types of measurements (TCS and CT). Only "modified" V3 diameters measured on CT using the angle correction were taken into account. For the Bland-Altman, the mean difference with $95 \% \mathrm{CI}$, the limits of agreement at 95\% CI were assessed [29]. The ICC and Bland-Altman plots were analysed separately for each side of measurement (right and left).

\section{Power analysis}

Expecting the V3 diameter measurements using TCS and CT to have an ICC of at least 0.85 based on previous data $[14-17,19]$ with a half range of the $95 \%$ CI of $0.15,59$ paired measurements of TCS and CT were necessary to analyse. Expecting 10\% of patients to have no V3 visible on TCS based on previous studies [15, 30], and expecting $10 \%$ to be excluded, we planned on including 100 patients.

\section{Diagnostic performance of V3 measurement using TCS to detect hydrocephalus}

Hydrocephalus was diagnosed by CT [26, 27]. The Mann-Whitney-Wilcoxon's test was used to compare V3 diameters between different subgroups of patients (patients with and patients without hydrocephalus). A $p$ value $<0.05$ was considered as significant. The risk of hydrocephalus was established from an analysis of statistical performances of the V3 TCS measurement, by the analysis of the receiver operating characteristic (ROC) curve. Using a previously published approach [31], we defined a grey zone (for which predicting hydrocephalus was not conclusive) for cut-offs with a sensitivity lower than $90 \%$ and a specificity lower than $90 \%$ (diagnosis tolerance of $10 \%)$. On either side of these cut-offs, patients were assigned to a low-risk or a high-risk.

\section{Absolute difference between V3 measurement by residents and experts (learning curve)}

The absolute difference between V3 diameters measured by residents and expert physicians using TCS was calculated. One millimetre of absolute difference between the measurements of the resident and the expert physician defined a satisfactory skill. The mean number of assessments needed to reach this threshold was recorded. Every subsequent measurement performed by a given resident was made on a different patient.

\section{Results}

Between August 2016 and May 2017, 100 consecutive adult patients were included. Additional file 1: Figure S2 shows the study flowchart. Table 1 and Additional file 1: Table S1 show patient characteristics and outcomes.

CT and TCS findings are shown in Table 2 and Additional file 1: Table S2. Hydrocephalus was reported in 35 patients. The middle cerebral artery was identified on TCS in 78 patients. Systolic, diastolic and mean cerebral artery velocities were $92 \mathrm{~cm} / \mathrm{s}$ (IQR 76-120), $28 \mathrm{~cm} / \mathrm{s}$ (IQR 22-40) and $48 \mathrm{~cm} / \mathrm{s}$ (IQR 37-64), respectively. The median pulsatility index (PI) was 1.2 (IQR 1.1-1.6). TCS examination including middle artery Doppler and V3 assessment was performed in 7 (IQR 5-10) min. Detailed data regarding Doppler measurements are shown Additional file 1: Table S2.

\section{Feasibility and reliability of TCS compared with brain CT (primary goal)}

The V3 diameter could be measured by TCS on at least one side in 87 patients $(87 \%)$ and on both sides in 70 patients (70\%). The V3 diameter could not be measured in 13 patients because of the impossibility to recognise the V3, in any side. "Modified" V3 diameters using the angle correction, measured by CT (gold standard), were 6.4 (IQR [4.4-9.2]) $\mathrm{mm}$ from the right side, and 6.3 (IQR [4.6-9.6]) $\mathrm{mm}$ from the left side. V3 diameters measured by TCS were 5.8 (IQR [4.6-8.4]) $\mathrm{mm}$ for the right side, and 5.8 (IQR 4.4-8.6]) $\mathrm{mm}$ for the left side.

The ICC between TCS and CT were 0.90 (95\% CI 0.84 to 0.93 ) for the right side and 0.92 (CI 0.88 to 0.95 ) for the left side (Additional file 1: Figure S3). Figure 2 shows the Bland-Altman analysis diagram. Mean difference between CT and TCS was $0.36 \mathrm{~mm}$ (standard deviation (SD) $1.52 \mathrm{~mm}, 95 \%$ limits of agreement (95\% LOA) $-2.7 \mathrm{~mm}$ to $3.3 \mathrm{~mm}$ ) for the right side, and $0.25 \mathrm{~mm}$ (SD $1.47 \mathrm{~mm}, 95 \% \mathrm{LOA}-2.7 \mathrm{~mm}$ to $3.1 \mathrm{~mm}$ ) for the left side. For the right side, the $95 \% \mathrm{CI}$ of the mean difference was 0.36 (0.02 to 0.7$) \mathrm{mm}$ and the $95 \%$ $\mathrm{CI}$ of the lower and upper limits of agreement were -2.7 $(-3.28$ to -2.12$) \mathrm{mm}$ and $3.3(2.72$ to 3.88$) \mathrm{mm}$, respectively. For the left side, $95 \%$ of the mean difference was 0.25 ( -0.08 to 0.58$) \mathrm{mm}$ and the $95 \% \mathrm{CI}$ of the lower and 
Table 1 Demographic and medical characteristics

\begin{tabular}{|c|c|}
\hline Characteristics upon admission to ICU & $N=100$ \\
\hline Age (years) & $62[52-70]$ \\
\hline $\operatorname{Sex}(F / M)$ & $52 / 48$ \\
\hline Body mass index $\left(\mathrm{kg} \mathrm{m}^{-2}\right)$ & $26[22-29]$ \\
\hline \multicolumn{2}{|l|}{ Reason for admission to the ICU } \\
\hline Aneurysmal subarachnoid haemorrhage, $n(\%)$ & $33(33 \%)$ \\
\hline Intracranial haematoma, $n(\%)$ & $30(30 \%)$ \\
\hline Stroke, $n(\%)$ & $13(13 \%)$ \\
\hline Head trauma, $n(\%)$ & $5(5 \%)$ \\
\hline Post-operative patients, $n$ (\%) & $15(15 \%)$ \\
\hline Meningoencephalitis & $2(2 \%)$ \\
\hline Cardiac arrest & $1(1 \%)$ \\
\hline Undetermined coma & $1(1 \%)$ \\
\hline Simplified Acute Physiological Score II & $40[31-53]$ \\
\hline Glasgow Coma Scale & $7[4-13]$ \\
\hline Fisher score ${ }^{*}, n=33$ & $4[3,4]$ \\
\hline International Severity Score score ${ }^{* *}, n=5$ & $32[26-38]$ \\
\hline Characteristics upon study enrolment & $N=100$ \\
\hline Time between ICU admission and enrolment, days & $2[1-5]$ \\
\hline \multicolumn{2}{|l|}{ Therapeutics, $n(\%)$} \\
\hline Invasive mechanical ventilation & $95(95 \%)$ \\
\hline Sedation & $78(78 \%)$ \\
\hline Analgesia & $78(78 \%)$ \\
\hline Vasopressors (norepinephrine) & $30(30 \%)$ \\
\hline Milrinone & $9[9 \%]$ \\
\hline Nimodipine & 28 [28\%] \\
\hline Clinical hypertensive symptoms, $n$ (\%) & $10(10 \%)$ \\
\hline Cushing reflex, $n(\%)$ & $3(3 \%)$ \\
\hline Anisocoria, $n(\%)$ & $3(3 \%)$ \\
\hline Mydriasis, $n(\%)$ & $4(4 \%)$ \\
\hline External ventricular derivation, $n$ (\%) & $37(37 \%)$ \\
\hline Craniotomy, n (\%) & $14(14 \%)$ \\
\hline Duration of mechanical ventilation, days & $17[7-28]$ \\
\hline ICU length of stay, days, $n(\%)$ & $21[9-37]$ \\
\hline RANKIN score at ICU discharge & $4[3-6]$ \\
\hline Mortality in ICU, $n(\%)$ & $25(25 \%)$ \\
\hline
\end{tabular}

Continuous data are expressed in median [25th-75th percentiles]

ICU intensive care unit

*Fisher score was calculated for the 33 patients with aneurysmal subarachnoid haemorrhage

**International Severity Score was calculated for the 5 patients with brain trauma

upper limits of agreement were $-2.7(-3.27$ to -2.13$)$ $\mathrm{mm}$ and 3.1 (2.53 to 3.67$) \mathrm{mm}$, respectively.

\section{Diagnostic performance of V3 measurement using TCS to detect hydrocephalus}

The V3 was assessable by TCS in 31 (89\%) patients among the 35 patients with hydrocephalus diagnosed using the CT. The median V3 diameter measured by TCS was significantly greater in the hydrocephalus group ( 8.8 (IQR 6.8 to 11.7$) \mathrm{mm}$ ) compared to the non-hydrocephalus group (5.0 (IQR 3.8 to 6.1 ) mm, $p<0.0001$ ) (Additional file 1: Figure S4). The ROC curve analysis for V3 diameter measured by TCS had an AUC of 0.91 [95\% CI $0.85-0.98$ ]. The best threshold of V3 diameter associated with hydrocephalus diagnosis was $6.25 \mathrm{~mm}$, corresponding to a specificity of 0.79 and a sensibility of 0.88 
Table 2 CT and TCS findings

\begin{tabular}{|c|c|c|}
\hline & CT & TCS \\
\hline Bone thickness, mm & $3[2.2-4]$ & NA \\
\hline Radiodensity, Hounsfield unit & 1204 [975-1377] & NA \\
\hline Time between TCS and CT, min & NA & $38(25-52)$ \\
\hline V3 assessable on both sides, $n$ (\%) & $100(\%)$ & $70(70 \%)$ \\
\hline V3 assessable on one side, $n$ (\%) & $100(\%)$ & $87(87 \%)$ \\
\hline \multicolumn{3}{|l|}{ Right side§ } \\
\hline V3 assessable, $n(\%)$ & $100(100 \%)$ & 79 (79\%) \\
\hline V3 diameter, mm & $6.4[4.4-9.2]$ & $5.8[4.6-8.4]$ \\
\hline Angle correction & $14[11-18]$ & NA \\
\hline V3 depth (bone to V3), mm & $65[62-69]$ & $72[68-76]$ \\
\hline \multicolumn{3}{|l|}{ Left side§ } \\
\hline V3 assessable, $n(\%)$ & $100(100 \%)$ & $78(78 \%)$ \\
\hline V3 diameter, mm & $6.3[4.6-9.6]$ & $5.8[4.4-8.6]$ \\
\hline Angle correction & $13[10-16]$ & NA \\
\hline V3 depth, mm & $65[62-68]$ & $71[68-75]$ \\
\hline \multicolumn{3}{|l|}{ Other CT findings } \\
\hline V3 coronal diameter, mm & $6.0[4.4-9.0]$ & NA \\
\hline V3 haematoma, $n(\%)$ & $23(23 \%)$ & NA \\
\hline Hydrocephalus, $n(\%)$ & $35(35 \%)$ & NA \\
\hline Hydrocephalus management, $n / N(\%)$ & $8 / 35(23 \%)$ & NA \\
\hline External ventricle drain reopening, $n$ & 3 & NA \\
\hline $\begin{array}{l}\text { External ventricle drain level adapta- } \\
\text { tion, } n\end{array}$ & 4 & NA \\
\hline External ventricle drain placement, $n$ & 1 & NA \\
\hline Medication, $n$ & 1 & NA \\
\hline
\end{tabular}

Continuous data are expressed in median [25th-75th percentiles] §V3 CT diameters were "modified" diameters to take into account the usual angulations of the ultrasound probe

The sum differs from 8 because one patient received several therapeutics CT computed-tomography; NA not applicable; TCS transcranial sonography; V3 third cerebral ventricle

(Fig. 3a). Figure 3b shows the two-graph ROC curves for sensitivity and specificity defining an inconclusive zone (grey zone). TCS was inconclusive (sensitivity or specificity less than $90 \%$ ) in $27 / 87$ patients (31\%) for a V3 diameter between 5.2 and $7.7 \mathrm{~mm}$. The diagnosis of hydrocephalus was thus excluded in 35 (40\%) patients with a V3 diameter $<5.2 \mathrm{~mm}$ and confirmed in 25 (29\%) patients with a V3 diameter $>7.7 \mathrm{~mm}$.

The V3 measurement from the right side was included for these analyses because right side was the most frequently assessable side. The left side was included when V3 was not assessable from right side.

\section{Absolute difference between V 3 measurement by residents and experts (secondary end point)}

Figure 4 shows the absolute difference between the V3 measurement by residents and experts, representing a learning curve for the ability to measure the V3 diameter using TCS. Forty-five measurements were performed on 31 patients by 9 residents. After 5 measurements, all residents reached a satisfactory measurement compared to the expert physician.

\section{Discussion \\ Main findings}

Feasibility was good, with 87 (87\%) of patients amenable to TCS measurements, and the reliability was high compared to the gold standard (brain CT). TCS was effective to exclude or confirm hydrocephalus in two-thirds of patients at bedside. Moreover, the learning curve is steep, with only 5 procedures necessary to acquire a satisfactory skill.

\section{Relation with existent literature}

Our findings are consistent with the existing literature concerning non-ICU patients. Regarding the feasibility of TCS to identify the V3, Bogdahn et al. [13] described V3 measurement in 45 of 52 subjects without neurological involvement (87\%). More recently, Schminke et al. [15] described V3 measurement in 27 of 29 subjects with multiple sclerosis (93\%). The V3 diameter was $4.4[ \pm 1.7] \mathrm{mm}$ in the study by Schminke et al. [15], while Puz et al. [32] found similar values with a median V3 diameter of 4.5 (IQR 3.8-5.4) $\mathrm{mm}$ in 20 healthy volunteers. In the present study, neuro-ICU patients had a median V3 diameter of $6.5 \mathrm{~mm}$ [4.4 to 9.1]. The larger V3 diameters in the present study may be explained by the differences in patients pathologies and ages, V3 enlarging with age [33]. The mean age of patients included in the present study was higher than for the patients included in the two previous studies [15, 32] (59 versus 37 years). Bendella et al. described similar V3 diameters than us in patients with a mean age of 56 years after a decompressive craniectomy [34].

V3 diameter measurement by TCS compared to CT, is consistent with the findings of the recent retrospective study by Oliveira et al. [19] that showed an ICC of 0.85 . However, in that study, the V3 diameters were greater than $30 \mathrm{~mm}$ and limits of agreement were very large (IQR -14.97 to $14.81 \mathrm{~mm}$ ). This could be explained by the retrospective nature of this study, the absence of a standardised measurement of the V3 by CT, as well as the small number of patients $(n=15)$. 
Right V3,diameters

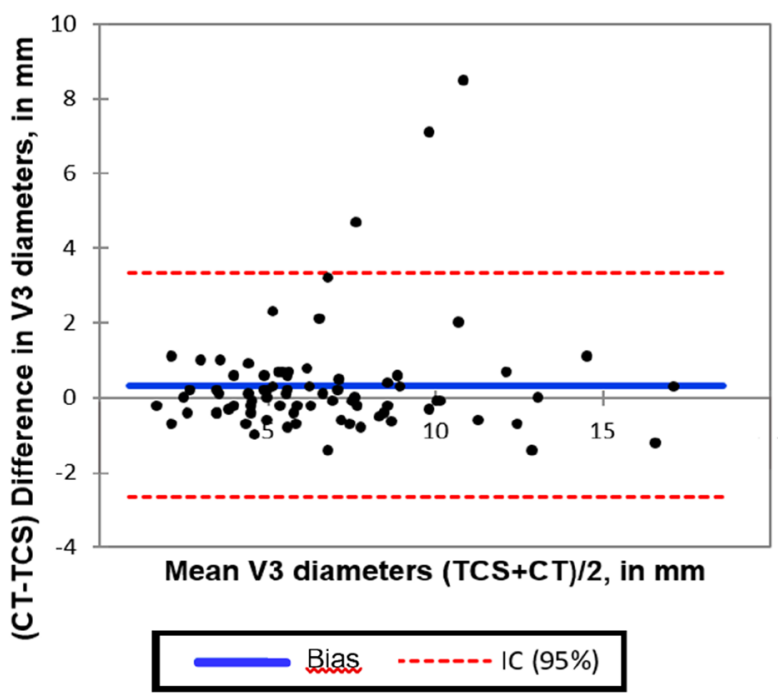

Left V3 diameters

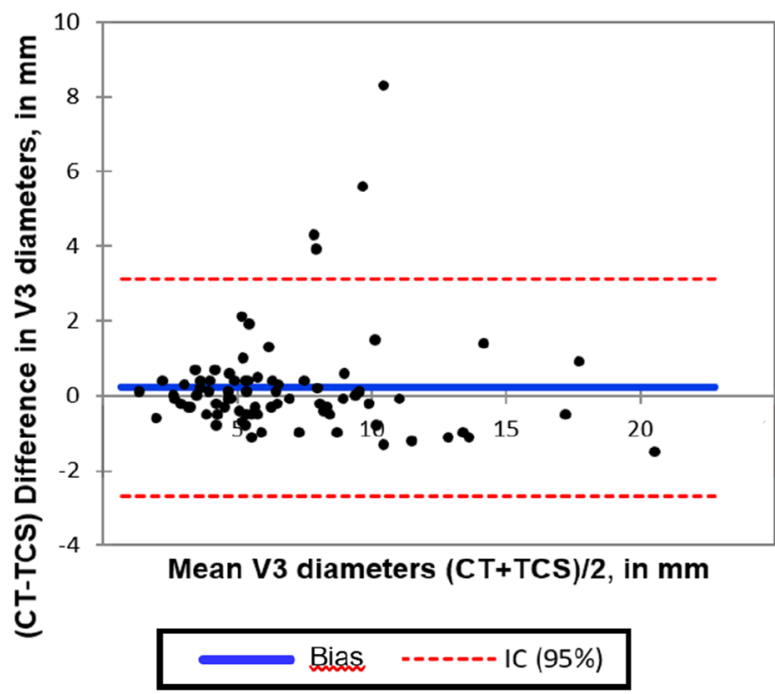

Fig. 2 V3 diameters measured by TCS and CT according to Bland-Altman diagram. Bland-Altman plots show the agreement between TCS and CT for V3 diameter measurements for each side of measurement. Bars lines represent $95 \%$ limits of agreement, and blue lines represent the mean difference. Mean difference between CT and TCS was $0.36 \mathrm{~mm}$ (standard deviation (SD) $1.52 \mathrm{~mm}, 95 \%$ limits of agreement (95\% LOA) - 2.7 mm to $3.3 \mathrm{~mm}$ ) for the right side, and $0.25 \mathrm{~mm}$ (SD $1.47 \mathrm{~mm}, 95 \% \mathrm{LOA}-2.7 \mathrm{~mm}$ to $3.1 \mathrm{~mm}$ ) for the left side

\section{Clinical implications}

TCS has a pivotal role in the ICU: it is a non-invasive, reproducible and bedside available tool, with a low cost. In patients with an adequate acoustic window (almost 9 patients out of 10), it allows for a non-irradiating assessment of the brain parenchyma and cerebral blood flow [35] without the risks of repeated CT scans, especially regarding the risk of transport and monitoring of patients to the CT room, as well as the risk of EVD disconnection related to the patient mobilisation [36].

The V3 diameter was measurable in 87 (87\%) of our patients and, compared to the CT, was conclusive in $60 / 87$ patients (69\%) for a V3 diameter $<5.2$ or $>7.7 \mathrm{~mm}$ for diagnosing hydrocephalus (Fig. 4). Therefore, TCS provides a reliable information on the diagnosis of hydrocephalus for 60 patients (60\%) and offers the clinician a follow-up of the V3 diameter, without the risk of repeated CT scans.

TCS could be helpful to monitor EVD management and weaning at the bedside, or to detect clinically occult hydrocephalus. EVD management impacts the ICU and hospital lengths of stay in patients with aneurysmal subarachnoid haemorrhage [37]. In 2016, the American Neurocritical Care Society released recommendations to encourage EVD weaning "as quickly as clinically feasible" [38]. Despite this recommendation, Chung et al. showed that EVD weaning was mainly gradual over 4 days in the USA [39]. During EVD weaning, patients may have rapid change of the V3 diameter. This is usually suspected when a clinical worsening is observed and confirmed only by brain $\mathrm{CT}$. The clinical examination during EVD weaning can be difficult, especially in unconscious ICU patients. Indeed, the neurological examination of sedated patients is challenging. Despite the fact that ICU patients are nowadays less sedated [40-43], one of the last indications for a deep or prolonged sedation is for brain injured patients [44]. According to the present study, TCS might allow an easy and safe screening of clinically occult hydrocephalus that can be performed systematically and repeatedly at the bedside. The present study showed that TCS can precisely detect patients with or without hydrocephalus in $69 \%$ of the cases when the V3 is assessable (Fig. 3). Further studies based on V3 measurement and TCS Doppler are necessary before implementing such a strategy determining the proportion of patients for whom TCS could shorten the time of recognition of hydrocephalus as well as the time to intervention. In many patients, a confirmation using $\mathrm{CT}$ is mandatory. TCS could also detect an early increase of the V3 diameter during EVD weaning. TCS provides an enhanced monitoring of brain injured patients and allows performing CT or therapeutic change quickly. In neurocritical care, time is brain, and a diminution of the duration of brain injury related to acute hydrocephalus could improve patient outcome. Such strategy remains to be evaluated by further studies. However, implementing V3 measurements by TCS 

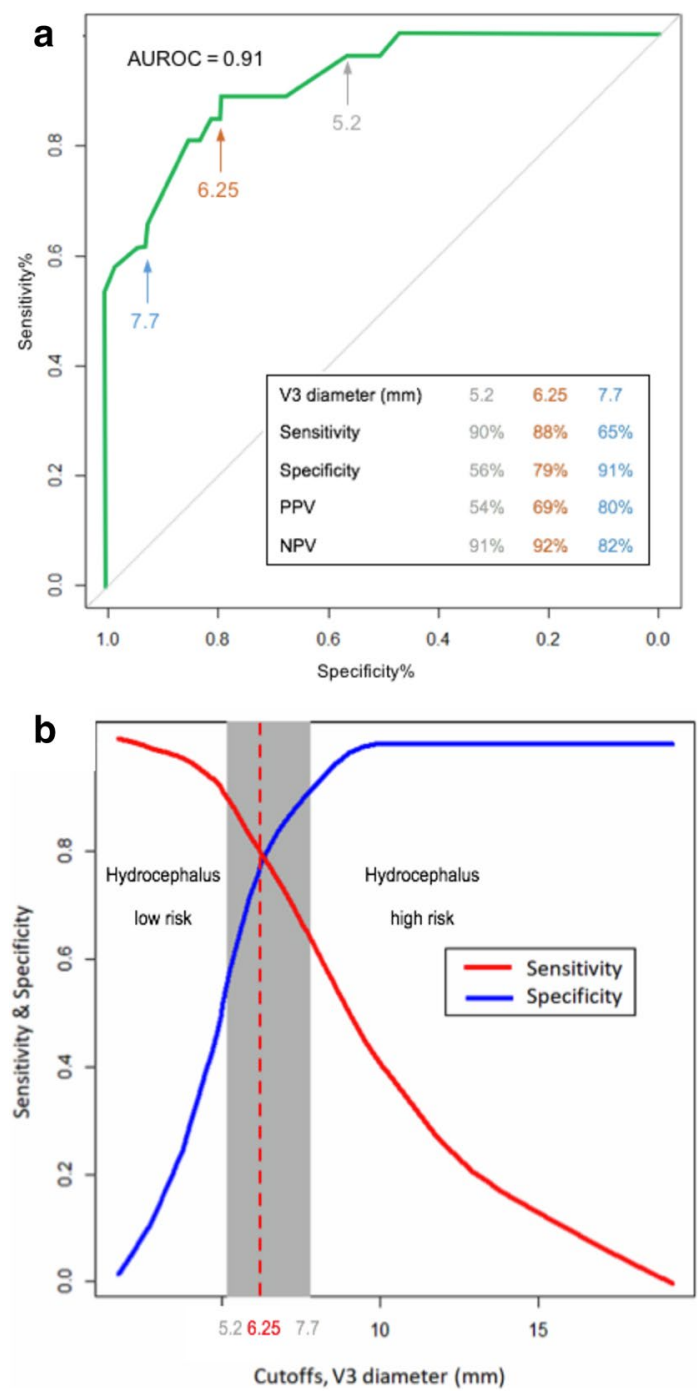

Fig. 3 a Receiver operating characteristic (ROC) curve of $\mathrm{V} 3$ diameter measured by TCS to detect hydrocephalus diagnosed on $\mathrm{CT}$. The ROC curve represents the specificity and sensitivity of TCS to diagnose hydrocephalus. AUC was $0.91 \%$ [95\% Cl 0.85-0.98\%], the estimated best threshold was at $6.25 \mathrm{~mm}$, with a specificity of 0.79 and a sensibility of 0.88 . Positive predictive value (PPV) and negative predictive value (NPV) are provided for thresholds on either side of the grey zone (see Fig. 3b). b Two-curve sensitivity/specificity representation of grey zone for $\mathrm{V} 3$ diameter measured by TCS, associated with a hydrocephalus diagnosed on CT. We defined a grey zone for cut-offs with a sensitivity lower than $90 \%$ and a specificity lower than 90\% (diagnosis tolerance of 10\%). On either side of these cut-offs, patients were assigned to a low-risk or a high-risk. The diagnosis of hydrocephalus was thus excluded in 35 (40\%) patients with a V3 diameter $<5.2 \mathrm{~mm}$ and confirmed in 25 (29\%) patients with a V3 diameter $>7.7 \mathrm{~mm}$. TCS was inconclusive in $27 / 87$ (31\%) patients. The $\mathrm{V} 3$ measurement from the right side was included for these analyses because right side was the most frequently assessable side. The left side was included when $\mathrm{V} 3$ was not assessable from right side requires the availability of this technique as well as a sufficient training. Other indications of TCS to localise cerebral ventricles in the future could be to help inserting DVE, but this procedure needs further investigation.

Every anaesthesiologist-intensivist resident with previous TCS Doppler experience but without V3 sonography experience could reach satisfactory skill after only five measurements. Four residents out of 9 made a substantial error on the third measurement. Therefore, even if the second measure is close to that of the expert operator, five measurements seem necessary to reach satisfactory skill. Measuring the V3 diameter by TCS appears to be an easy skill to learn. This tool could become widespread over ICUs after a short training by expert TCS operators. If the relevance of V3 monitoring is confirmed, it could be implemented as a first line strategy and interest centres where brain $\mathrm{CT}$ is not quickly available.

Strengths of this study are that it is the first prospective study, in a large population of neuro-ICU patients with diverse brain illnesses, which compared TCS and CT on V3 measurements, each investigator blinded to one other, with standardised measurements for both TCS and CT. Furthermore, the study had a high inclusion rate of consecutively admitted patients within a short period of time ( 8 months). This suggests the feasibility of TCS in routine.

Our study has several limitations. First, we were unable to measure V3 diameter with TCS in 13 patients (13\%) because of the impossibility to recognise the V3 using echography. The risk of failure using echography in critically ill patients is one of the main issues related to this technique, as also describe for other indications such as cardiac (1\% of failure [45]) or pneumothorax (12\% of failure [46]) assessments. Secondly, we did not directly assess intra- and inter-observer reliabilities. Regarding the intra-observer reliability, it can be estimated comparing measurements on both sides in the 70 patients with bilateral V3 visualisation. The ICC was 98\% suggesting a good intra-observer reliability (Additional file 1: Figure S5). These findings are consistent with the literature, Schminke et al. showing an intra-observer ICC of $99.4 \%$ [15]. Regarding the inter-observer reliability, the study was not designed to test this property, but to assess the training of residents. The learning curves could reflect indirectly inter-observer reliability that was good after only a short training period. In all, V3 diameter measurement by TCS seems to be a reliable tool. Finally, we could not compare the time taken by the residents to the time taken by the experts to perform the measures. This is because the time that was measured for the experts included the V3 measurement as well as the Doppler velocities (accuracy study). To measure the V3 diameter, the residents took a median of 7 (IQR [5 to 8]) min 


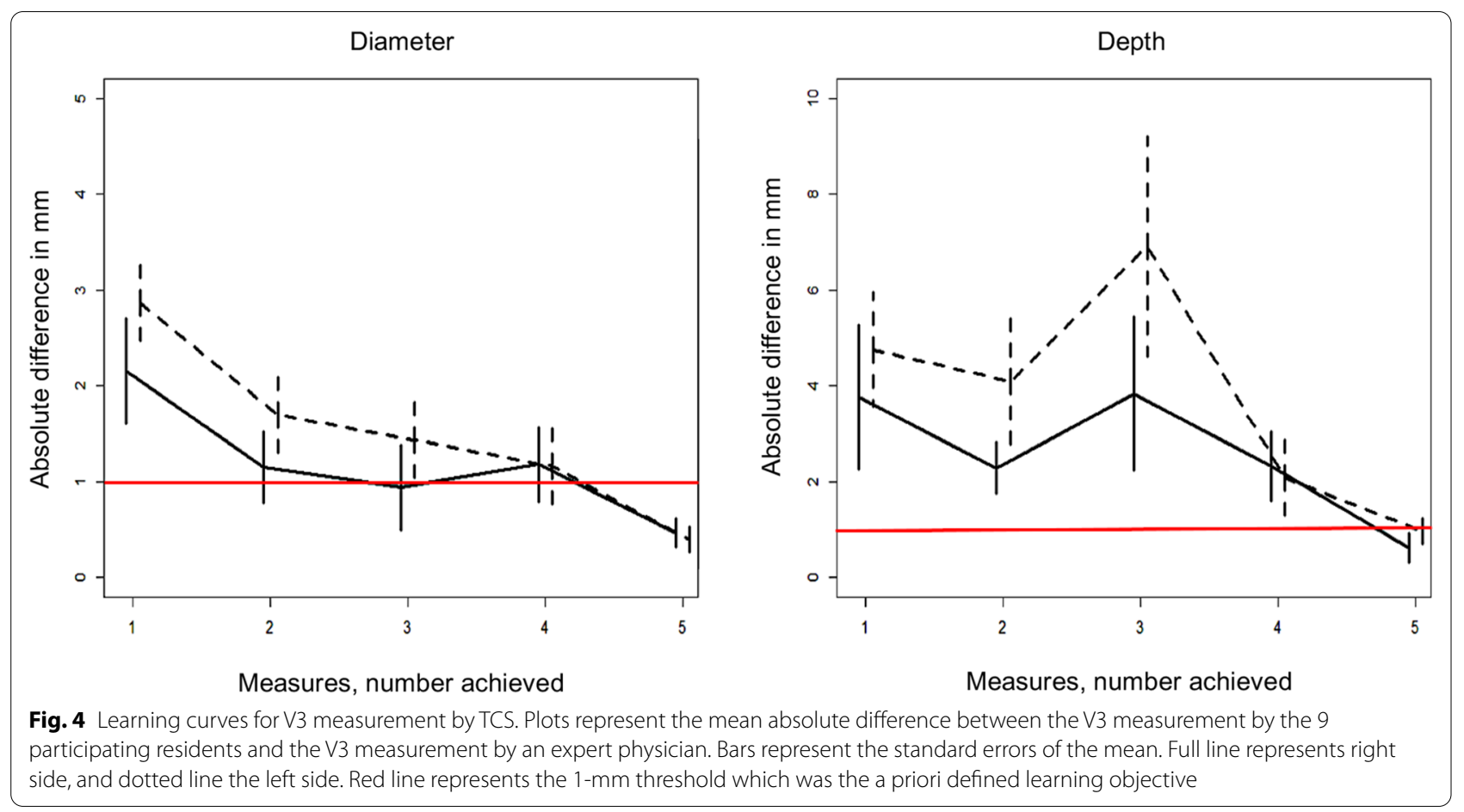

and 5 (IQR [3 to 7]) min at their first and last attempt, respectively, suggesting a skill improvement (learning curve).

In conclusion, TCS is a feasible, simple, reliable and non-invasive tool for measuring V3 diameters in neuroICU patients at the bedside and is associated with a short learning curve. Monitoring V3 diameters by TCS could be a point-of-care tool to facilitate the early diagnosis of hydrocephalus. The role of TCS for the measurement of the V3 diameter in the management and weaning of EVD requires further studies.

\section{Abbreviations}

ICU: Intensive care unit; TCS: Transcranial sonography; CT: Computed-tomography; V3: Third ventricle; ICC: Intraclass correlation coefficient; APS2: Simplified Acute Physiology Score 2; ISS: International Severity Score; EVD: External ventricular drain; IQR: Interquartile range; 95\% Cl: 95\% Confidence interval; ROC: Receiver operating characteristic; PI: Pulsatility index.

\section{Supplementary Information}

The online version contains supplementary material available at https://doi. org/10.1186/s13613-021-00857-x.

Additional file1: Table S1: Patient characteristics, Table S2: CT and TCS findings for the 100 patients included for analysis, Figure S1: Study design, Figure S2: Study flow chart, Figure S3: Right and left sides V3 diameters measured by TCS compared with CT, Figure S4: Comparison of V3 diameters measured by TCS, according to the hydrocephalus status, Figure S5: V3 diameters measured by TCS compared with the other side

\section{Acknowledgements}

We specifically thank Christopher Scott, MD, for having substantially edited the manuscript as a native English speaker.

\section{Authors' contributions}

RW, FG, PFP, NM and GC designed the study. RW, PB, FG, FP, AM and FD performed the V3 TCS measurements. NM performed the statistical analysis. RW performed all blind CT measurements. RW, NM and GC drafted the manuscript. All authors read and approved the final manuscript.

\section{Funding}

Author's own work.

\section{Availability of data and materials}

The datasets used and analysed during the current study are available from the corresponding author on reasonable request. Preliminary data of this study were presented at the annual meeting of the French Society of Anaesthesia and Intensive Care Medicine, 21-23 September 2017, Paris.

\section{Declarations}

Ethics approval and consent to participate

The study protocol was approved by the Ethics committee of "Comité de Protection des Personnes Sud-Méditerranée I" (ID RCB: 2016-A00749-42; Protocol Version: March 11, 2016; consent version: June 25, 2016), ClinicalTrials.gov ID: NCT02830269. Because the trial used non-invasive procedures along with standard care provided in French ICUs, only a verbal consent was required from the patient or relatives, according to the French law.

\section{Consent for publication}

Not applicable.

\section{Competing interests}

The authors declare that they have no competing interests.

\section{Author details}

${ }^{1}$ Department of Anaesthesia \& Critical Care Medicine, Montpellier University Hospital Center, Gui de Chauliac Hospital, Montpellier, France. ${ }^{2}$ Department 
of Anaesthesia \& Critical Care Medicine, Montpellier University Hospital Center, Saint Eloi Hospital, Montpellier, France. ${ }^{3}$ Department of Statistics, Montpellier University Hospital Center, La Colombière Hospital, and Institut Montpelliérain Alexander Grothendieck (IMAG), University of Montpellier, CNRS, Montpellier, France. ${ }^{4}$ PhyMedExp, University of Montpellier, INSERM, CNRS, Montpellier, France.

Received: 14 January 2021 Accepted: 21 April 2021

Published online: 04 May 2021

\section{References}

1. Rasulo FA, Togni T, Romagnoli S. Essential noninvasive multimodality neuromonitoring for the critically ill patient. Crit Care. 2020;24:100.

2. Oddo M, Bracard S, Cariou A. Update in neurocritical care: a summary of the 2018 Paris international conference of the French Society of Intensive Care. Ann Intensive Care. 2019:9:47.

3. Bederson JB, Connolly ES, Batjer HH, Dacey RG, Dion JE, Diringer MN, et al. Guidelines for the management of aneurysmal subarachnoid hemorrhage a statement for healthcare professionals from a special writing group of the Stroke Council American Heart Association. Stroke. 2009:40:994-1025.

4. Carney N, Totten AM, O'Reilly C, Ullman JS, Hawryluk GWJ, Bell MJ, et al. Guidelines for the management of severe traumatic brain injury fourth edition. Neurosurgery. 2017;80:6-15.

5. Jauch EC, Saver JL, Adams HP, Bruno A, Connors Buddy JJ, Demaerschalk BM, et al. Guidelines for the early management of patients with acute ischemic stroke a guideline for healthcare professionals from the American Heart Association/American Stroke Association. Stroke. 2013:44:870-947.

6. Sloan MA, Haley EC, Kassell NF, Henry ML, Stewart SR, Beskin RR, et al. Sensitivity and specificity of transcranial Doppler ultrasonography in the diagnosis of vasospasm following subarachnoid hemorrhage. Neurology. 1989;39:1514.

7. Velly LJ, Bilotta F, Fàbregas N, Soehle M, Bruder NJ, Nathanson MH, et al. Anaesthetic and ICU management of aneurysmal subarachnoid haemorrhage: a survey of European practice. Eur J Anaesthesiol. 2015;32:168-76.

8. Ract C, Le Moigno S, Bruder N, Vigué B. Transcranial Doppler ultrasound goal-directed therapy for the early management of severe traumatic brain injury. Intensive Care Med. 2007;33:645-51.

9. Bhuiyan MR, Deb S, Mitchell RA, Teddy PJ, Drummond KJ. The effect of formal training on the clinical utility of transcranial Doppler ultrasound monitoring in patients with aneurysmal subarachnoid haemorrhage. J Clin Neurosci. 2012;19:1255-60.

10. Rasulo FA, Bertuetti R, Robba C, Lusenti F, Cantoni A, Bernini M, et al. The accuracy of transcranial Doppler in excluding intracranial hypertension following acute brain injury: a multicenter prospective pilot study. Crit Care. 2017;21:44.

11. Robba C, Pozzebon S, Moro B, Vincent J-L, Creteur J, Taccone FS. Multimodal non-invasive assessment of intracranial hypertension: an observational study. Crit Care. 2020;24:379.

12. Robba C, Goffi A, Geeraerts T, Cardim D, Via G, Czosnyka M, et al. Brain ultrasonography: methodology, basic and advanced principles and clinical applications a narrative review. Intensive Care Med. 2019;45:913-27.

13. Bogdahn U, Becker G, Winkler J, Greiner K, Perez J, Meurers B. Transcranial color-coded real-time sonography in adults. Stroke. 1990;21:1680-8.

14. Kallmann B-A, Sauer J, Schließer M, Warmuth-Metz M, Flachenecker P, Becker Dagger $G$, et al. Determination of ventricular diameters in multiple sclerosis patients with transcranial sonography (TCS): a two year followup study. J Neurol. 2004;251:30-4.

15. Schminke U, Lorenz L, Kirsch M, Von Sarnowski B, Khaw AV, Kessler C, et al. Diameter assessment of the third ventricle with transcranial sonography in patients with multiple sclerosis. J Neuroimaging. 2010;20:53-7.

16. Berg D, Mäurer M, Warmuth-Metz M, Rieckmann P, Becker G. The correlation between ventricular diameter measured by transcranial sonography and clinical disability and cognitive dysfunction in patients with multiple sclerosis. Arch Neurol. 2000:57:1289-92.

17. Sahuquillo P, Tembl JI, Parkhutik V, Vázquez JF, Sastre I, Lago A. The study of deep brain structures by transcranial duplex sonography and imaging resonance correlation. Ultrasound Med Biol. 2013;39:226-32.
18. Mursch K, Vogelsang JP, Zimmerer B, Ludwig HC, Behnke J, Markakis E. Bedside measurement of the third ventricle's diameter during episodes of arising intracranial pressure after head trauma. Acta Neurochir. 1995;137:19-24.

19. Oliveira RAG, de Oliveira LM, Paiva WS, de Sá Malbouisson LM, Teixeira MJ, Bor-Seng-Shu E. Comparison between brain computed tomography scan and transcranial sonography to evaluate third ventricle width, peri-mesencephalic cistern, and sylvian fissure in traumatic brain-injured patients. Front Neurol. 2017:8:44

20. Leone M, Constantin J-M, Dahyot-Fizelier C, Duracher-Gout C, JoannesBoyau O, Langeron O, et al. French intensive care unit organisation. Anaesth Crit Care Pain Med. 2018;37:625-7.

21. Toulouse E, Lafont B, Granier S, McGurk G, Bazin J-E. French legal approach to clinical research. Anaesth Crit Care Pain Med. 2020;39:883-5.

22. Le Gall JR, Lemeshow S, Saulnier F. A new simplified acute physiology score (SAPS II) based on a European/North American multicenter study. JAMA. 1993;270:2957-63.

23. Fisher CM, Kistler JP, Davis JM. Relation of cerebral vasospasm to subarachnoid hemorrhage visualized by computerized tomographic scanning. Neurosurgery. 1980;6:1-9.

24. Baker SP, O'Neill B, Haddon W, Long WB. The injury severity score: a method for describing patients with multiple injuries and evaluating emergency care. J Trauma. 1974;14:187-96.

25. Talairach J, Tournoux P. Practical examples for the use of the atlas in neuroradiologic examinations. In: Co-planar stereotaxic atlas of the human brain: 3-Dimensional proportional system: an approach to cerebral imaging. New York: Thieme Medical Publishers, Inc.; 1988.

26. Bewick V, Cheek L, Ball J. Statistics review 13: receiver operating characteristic curves. Crit Care. 2004;8:508-12.

27. Dupont S, Rabinstein AA. CT evaluation of lateral ventricular dilatation after subarachnoid hemorrhage: baseline bicaudate index values [correction of values]. Neurol Res. 2013;35:103-6.

28. Bland JM, Altman DG. Measuring agreement in method comparison studies. Stat Methods Med Res. 1999:8:135-60.

29. Kottner J, Audigé L, Brorson S, Donner A, Gajewski BJ, Hróbjartsson A, et al. Guidelines for reporting reliability and agreement studies (GRRAS) were proposed. J Clin Epidemiol. 2011;64:96-106.

30. Hernández NL, Escrivá AG. Jordà JMM [Study of the diameter of the third ventricle with transcranial sonography]. Neurol Barc Spain. 2007;22:507-10.

31. Cannesson M, Le Manach Y, Hofer CK, Goarin JP, Lehot J-J, Vallet B, et al. Assessing the diagnostic accuracy of pulse pressure variations for the prediction of fluid responsiveness: a "gray zone" approach. Anesthesiology. 2011;115:231-41.

32. Puz P, Lasek-Bal A, Radecka P. Transcranial sonography of subcortical structures in patients with multiple sclerosis. Acta Neurol Scand. 2017;136:24-30.

33. Meese W, Kluge W, Grumme T, Hopfenmüller W. CT evaluation of the CSF spaces of healthy persons. Neuroradiology. 1980;19:131-6.

34. Bendella H, Maegele M, Hartmann A, Spreer J, Rommel N, Lefering R, et al. Cerebral ventricular dimensions after decompressive craniectomy: a comparison between bedside sonographic duplex technique and cranial computed tomography. Neurocrit Care. 2017:26:321-9.

35. Robba C, Cardim D, Sekhon M, Budohoski K, Czosnyka M. Transcranial Doppler: a stethoscope for the brain-neurocritical care use. J Neurosci Res. 2017. https://doi.org/10.1002/jnr.24148.

36. Caricato A, Pitoni S, Montini L, Bocci MG, Annetta P, Antonelli M. Echography in brain imaging in intensive care unit: State of the art. World J Radiol. 2014;6:636-42.

37. Klopfenstein JD, Kim LJ, Feiz-Erfan I, Hott JS, Goslar P, Zabramski JM, et al. Comparison of rapid and gradual weaning from external ventricular drainage in patients with aneurysmal subarachnoid hemorrhage: a prospective randomized trial. J Neurosurg. 2004;100:225-9.

38. Fried HI, Nathan BR, Rowe AS, Zabramski JM, Andaluz N, Bhimraj A, et al. The insertion and management of external ventricular drains: an evidence-based consensus statement: a statement for healthcare professionals from the Neurocritical Care Society. Neurocrit Care. 2016;24:61-81.

39. Chung DY, Leslie-Mazwi TM, Patel AB, Rordorf GA. Management of external ventricular drains after subarachnoid hemorrhage: a multiinstitutional survey. Neurocrit Care. 2017;26:356-61. 
40. Chanques G, Jaber S, Barbotte E, Violet S, Sebbane M, Perrigault P-F, et al. Impact of systematic evaluation of pain and agitation in an intensive care unit. Crit Care Med. 2006;34:1691-9.

41. Mehta S, Burry L, Cook D, Fergusson D, Steinberg M, Granton J, et al. Daily sedation interruption in mechanically ventilated critically ill patients cared for with a sedation protocol: a randomized controlled trial. JAMA. 2012;308:1985-92.

42. Chanques G, Conseil M, Roger C, Constantin J-M, Prades A, Carr J, et al. Immediate interruption of sedation compared with usual sedation care in critically ill postoperative patients (SOS-ventilation): a randomised, parallel-group clinical trial. Lancet Respir Med. 2017;5:795-805.

43. Stephens RJ, Dettmer MR, Roberts BW, Ablordeppey E, Fowler SA, Kollef $\mathrm{MH}$, et al. Practice patterns and outcomes associated with early sedation depth in mechanically ventilated patients: a systematic review and metaanalysis. Crit Care Med. 2018;46:471-9.
44. Oddo M, Crippa IA, Mehta S, Menon D, Payen J-F, Taccone FS, et al. Optimizing sedation in patients with acute brain injury. Crit Care. 2016;20:128.

45. Joseph MX, Disney PJS, Da Costa R, Hutchison SJ. Transthoracic echocardiography to identify or exclude cardiac cause of shock. Chest. 2004;126:1592-7.

46. Lichtenstein DA, Mezière GA. Relevance of lung ultrasound in the diagnosis of acute respiratory failure: the BLUE protocol. Chest. 2008;134:117-25.

\section{Publisher's Note}

Springer Nature remains neutral with regard to jurisdictional claims in published maps and institutional affiliations.

\section{Submit your manuscript to a SpringerOpen ${ }^{\circ}$ journal and benefit from:}

- Convenient online submission

- Rigorous peer review

- Open access: articles freely available online

- High visibility within the field

- Retaining the copyright to your article

Submit your next manuscript at springeropen.com 